\title{
The significance of postpericardiotomy syndrome: A real threat or a simple nuisance?
}

\author{
Juan N. Pulido, MD
}

\footnotetext{
From the Cardiovascular Intensive Care Unit, Cardiothoracic Anesthesiology and Critical Care Medicine, Swedish Heart and Vascular Institute, Physicians Anesthesia Services, Seattle, Wash. Disclosures: Author has nothing to disclose with regard to commercial support.

Received for publication Nov 21, 2016; accepted for publication Nov 28, 2016; available ahead of print January $25,2017$.

Address for reprints: Juan N. Pulido, MD, Medical Director Cardiovascular Intensive Care Unit, Cardiothoracic Anesthesiology and Critical Care Medicine, Swedish Heart and Vascular Institute, Physicians Anesthesia Services, 550 17th Ave \#680, Seattle, WA 98122 (E-mail: juan.pulido@swedish.org).

J Thorac Cardiovasc Surg 2017;153:886-7

$0022-5223 / \$ 36.00$

Copyright (c) 2016 by The American Association for Thoracic Surgery

http://dx.doi.org/10.1016/j.jtcvs.2016.11.051
}

Postpericardiotomy syndrome (PPS) is a frequent complication after cardiac surgery that is well known but poorly understood. As such, there is no definite consensus on diagnosis and there is lack of clarity on its prognostic significance. The first reports were described in parallel to the origins of cardiac surgery ${ }^{1}$ and since then, it has been a familiar entity to the cardiac surgeon and perioperative cardiac care provider. Little is known, however, about risk factors and prognosis of these patients.

In this issue of the Journal, van Osch and colleagues ${ }^{2}$ sought to evaluate these very questions in a subgroup analysis from the large DECS (Intraoperative High Dose Dexamethasone for Cardiac Surgery) trial. ${ }^{3}$ The DECS trial was a double-blind, randomized, placebocontrolled trial investigating the effect of an intraoperative dose of $1 \mathrm{mg} / \mathrm{kg}$ dexamethasone on a composite endpoint of death, myocardial infarction, stroke, renal failure, or respiratory failure in 4494 adult patients undergoing cardiac surgery. ${ }^{3}$ The authors studied a subgroup of 822 patients who underwent valvular surgery because of the availability of postoperative echocardiography in all patients at days 4 to 6. PPS was defined the presence of 2 of 5 of the following: pericardial rub, fever $>72$ hours postoperatively, pleuritic chest pain, new significant pleural effusion on chest radiograph, or significant pericardial effusion $(\geq 10 \mathrm{~mm})$. The primary outcome of the study was reoperation for tamponade. Long-term secondary outcomes at 1 year were mortality, stroke, myocardial infarction, and readmissions (for any reason). Short-term secondary outcomes included hospital length of stay (days) and at 1 month, the incidence of postoperative atrial fibrillation, reoperation for surgical bleeding, or percutaneous pericardial or pleural drainage. There was a standard postoperative anticoagulation regimen and chest tube removal. The incidence of PPS was found to be $14.5 \%$ (similar to other series). After multivariate analysis, greater body mass index was associated with lower incidence

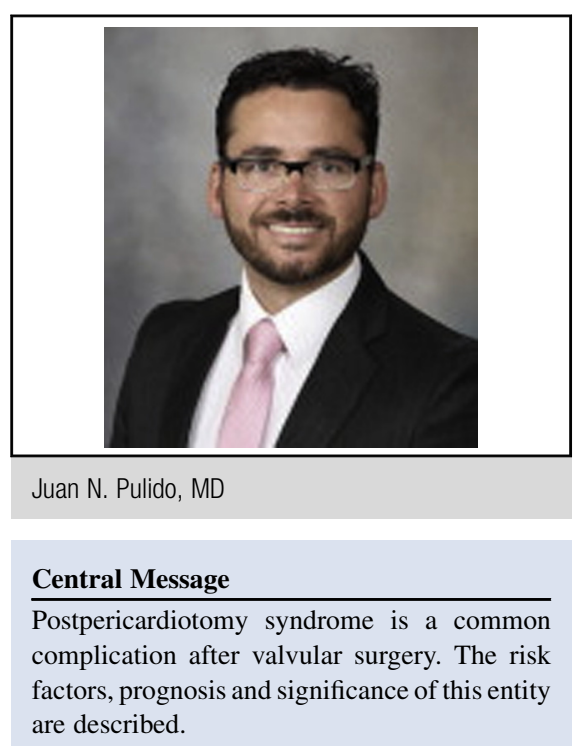

See Article page 878

of PPS and pulmonary disease not treated with corticosteroids was associated independently with PPS. The need for reoperation for tamponade within 1 year was $20.9 \%$ in patients who developed PPS versus $2.5 \%$ in patients without PPS. Patients with PPS also had longer hospital stays (13 vs 11 days). Nevertheless, there was no difference in postoperative atrial fibrillation, mortality, stroke, myocardial infarction, and readmissions. Moreover, there was no difference in baseline dexamethasone use in patients with and without PPS.

This study provides further evidence that PPS is an important complication after cardiac surgery despite no impact in mortality and major medical morbidity. PPS clearly is associated with longer hospital stay and greater risk of tamponade requiring reoperation. The lack of protective effect of dexamethasone as well as chronic steroid use questions the efficacy of treatment with steroids for the prevention of PPS. The significance of pulmonary disease and body mass index on the development of this entity remains unclear. Important aspects that were not evaluated in this study but remain relevant are the fact that it included only valvular surgery and the lack of detail in some surgical aspects such as pericardial closure, all due to the retrospective nature of the study. It is also important to note that 1 year was probably not enough follow-up to rule out PPS as a possible contributor for development of constrictive pericarditis. 


\section{References}

1. Ito T, Engle MA, Goldberg HP. Postpericardiotomy syndrome following surgery for non-rheumatic heart disease. Circulation. 1958;17:549-56.

2. van Osch D, Dieleman JM, Bunge JJ, van Dijk D, Doevedans PA, Suyker WJ, et al. Risk factors and prognosis of the postpericardiotomy syndrome in

patients undergoing valve surgery. J Thorac Cardiovasc Surg. 2017;153: 878-85.

3. Dieleman JM, Nierich AP, Rosseel PM, van der Maaten JM, Hofland J, Diephuis JC, et al. Intraoperative high dose dexamethasone for cardiac surgery: a randomized controlled trial. JAMA. 2012;308:1761-7. 